\section{Update on complications in pediatric anesthesia}

\author{
Giovanni de Francisci, ${ }^{1}$ \\ Angela Elisa Papasidero,1 \\ Giorgia Spinazzola, ${ }^{1}$ Dario Galante, 2 \\ Marco Caruselli, 3 Dino Pedrotti, 4 \\ Antonio Caso, 5 Massimo Lambo, 6 \\ Matteo Melchionda,2 \\ Maria Grazia Faticato 7
}

1Department of Anesthesiology and Intensive Care Agostino Gemelli Hospital, Catholic University of the Sacred Heart, Rome; 2University Department of Anesthesia and Intensive Care, University Hospital Ospedali Riuniti, Foggia;

3Department of Anesthesia and Intensive Care, Salesi Children's Hospital, Ancona;

4Department of Anesthesia and Intensive Care, S. Chiara Hospital, Trento;

5Department of Anesthesia and Intensive Care, Casa Sollievo della Sofferenza

Hospital, San Giovanni Rotondo;

6Department of Anesthesia and Intensive

Care, SS. Annunziata Hospital, Sulmona;

7 University Department of Pediatric

Surgery, University Hospital Ospedali

Riuniti, Foggia, Italy

\section{Abstract}

Complications in pediatric anesthesia can happen, even in our modern hospitals with the most advanced equipment and skilled anesthesiologists. It is important, albeit in a tranquil and reassuring way, to inform parents of the possibility of complications and, in general, of the anesthetic risks. This is especially imperative when speaking to the parents of children who will be operated on for minor procedures: in our experience, they tend to think that the anesthesia will be a light anesthesia without risks. Often the surgeons tell them that the operation is very simple without stressing the fact that it will be done under general anesthesia which is identical to the one we give for major operations. Different is the scenario for the parents of children who are affected by malignant neoplasms: in these cases they already know that the illness is serious. They have this tremendous burden and we choose not to add another one by discussing anesthetic risks, so we usually go along with the examination of the child without bringing up the possibility of complications, unless there is some specific problem such as a mediastinal mass.

\section{Introduction}

The pre-anesthetic evaluation is of great importance for pediatric patients, particularly in neonatal and preterm infant anesthesia. The preterm infant (born before 37 weeks of gestation) is at the highest level of risk for surgery and anesthesia, especially if the body weight is very low (below $2000 \mathrm{~g}$ ). In the preterm infant the surfactant concentration is low, and this entails a reduction in lung volume and compliance, with an increase in intrapulmonary shunt and ventilation/perfusion mismatch. If there is a reduction, for whatever reason, in lung volume, the intrapulmonary shunt worsens. On the other end, the preterm baby is susceptible to oxygen toxicity and pulmonary barotrauma.

The pre-anesthetic evaluation of the child is very important for all cases. We rely on the clinical examination, on the personal and medical anamnestic data, and do not perform any laboratory investigation unless some indication arises from the examination. Previous anesthetic records are of utmost importance for information on problems encountered by our colleagues.

The induction of anesthesia is a magical moment, and one in which problems can arise. The operating room must be warmed and the ambiance must be comfortable, free of noise and loud conversation. A pre-induction checklist is advisable to avoid yelling requests for items while slowly inducing the anesthesia: during the induction we usually tell a story, or softly sing a song, and this procedure must go uninterrupted.

\section{Complications related to drugs}

0xygen is a very important gas. We all know that without it life is impossible, but we at the same time must remember that oxygen is a medical gas, a drug, and it has its own side effects. So, while doing all we can to prevent hypoxia, we have to keep the oxygen concentration of the inspired gas in the anesthesia machine at the lowest level to remain compatible with a good hemoglobin saturation. High oxygen concentrations can dilute the nitrogen content of lung regions and can promote absorption atelectasis; 1,2 high flows of dry $0 x y-$ gen can dry and irritate mucosal surfaces of the air passages as well as decrease mucociliary transport and clearance of secretions. ${ }^{2}$ Oxygen in excess causes increased production of hydrogen peroxide, superoxide anion, singlet oxygen and hydroxyl radicals, which are toxic to lipids and proteins in biological membranes. In premature babies excess oxygen can provoke eye injury such as retrolental
Correspondence: Giovanni de Francisci, Department of Anesthesiology and Intensive Care Agostino Gemelli Hospital, Catholic University of the Sacred Heart, Rome, Italy.

E-mail: giovannigdf50@gmail.com

Key words: pediatric anesthesia, complications, anesthetic drugs, preanesthetic evaluation.

Conflict of interests: the authors declare no potential conflict of interests.

Received for publication: 15 October 2012. Revision received: 30 November 2012. Accepted for publication: 1 February 2013.

This work is licensed under a Creative Commons Attribution NonCommercial 3.0 License (CC BYNC 3.0).

(C) Copyright G. de Francisci et al., 2013 Licensee PAGEPress, Italy

Pediatric Reports 2013; 5:e2 doi:10.4081/pr.2013.e2

fibroplasia. Finally, any oxygen-enriched atmosphere constitutes a fire hazard.2

Inhalation anesthetic agents can depress the myocardium so we always add opioids to deepen the anesthetic plan without relying on only to volatile anesthetics. Regional techniques such as caudal block and inguinal block can also be valuable in this regard. It is important to remember that inhalational anesthetics have protective effects against ischemia-reperfusion injury, and recent authors suggest that these effects may also result in clinically relevant improved organ function. ${ }^{3}$ This protective capacity has been related to a preconditioning effect, a postconditioning effect, and also to an effect on apoptosis. With the currently used volatile agents (Sevoflurane, Desflurane) anesthetic hepatotoxicity is no longer a significant clinical problem. ${ }^{3}$ Sevoflurane and Desflurane are contraindicated only in the very rare cases of malignant hyperthermia susceptibility. Sevoflurane is the ideal agent for inhalation induction of anesthesia in children because it is not irritating to the airway. ${ }^{4} \mathrm{We}$ use up to $8 \%$ of sevoflurane for induction, promptly decreasing the dialed concentration as soon as the child loses consciousness. Sevoflurane can react with desiccated $\mathrm{CO} 2$ absorbent (i.e., an absorbent in which a prolonged gas flow has passed for a long time) and can undergo an exothermic reaction, damaging the airways. ${ }^{4}$ Sevoflurane is a very effective bronchodilator, and, despite early controversial reports about nephrotoxicity by means of the production of Compound A, a degradation product resulting from the interaction of sevoflurane with the $\mathrm{CO} 2$ absorbent soda lime, it is actually not at all toxic to the kidney as long as a fresh gas flow is kept at no less than 
2 litres per minute. ${ }^{4}$ Desflurane is not suitable for inhalation induction due to its irritating effect on the airways, but we use it for the maintenance of anesthesia (after tracheal intubation) even in newborns. Desflurane is a bronchodilator and has a modest negative inotropic effect; it does not have any nephrotoxic effect. Nitrous oxide can inactivate vitamin B12, provoking neurologic disorders both in patients and in operating room personnel. We never use nitrous oxide. Toxic effects are particularly possible in persons with a subclinical, preexisting deficit of vitamin B12 but it is not possible to know in advance which patients are deprived of vitamin B12. Nitrous oxide also has the well-known effect of expanding any air-filled cavity.

Neuromuscular blockers are the most useful drugs in our pharmacologic armamentarium but postanesthetic morbidity associated with incomplete reversal of neuromuscular blockers is still a frequent occurrence. Sugammadex, a relatively new drug, is a cyclodextrin that can form a very tight complex with rocuronium (less with vecuronium and pancuronium) thus allowing a reversal even during a profound neuromuscular block. ${ }^{5}$ Cysteine can reverse the effects of Gantacurium, a new relaxant that is an asymmetric mixed-onium chlorofumarate, and of AV002, an investigational nondepolarizing neuromuscular blocking drug, 5 but there are still doubts about the safety of giving exogenous cysteine. Suxamethonium, the only depolarizing muscle relaxant, has various side effects: it is a trigger for malignant hyperthermia, can provoke hyperkalemia, rhabdomyolysis, masseter spasms and its action can be very prolonged in individuals with low blood levels of cholinesterases. We never use suxamethonium: in cases in which we want a rapid onset of paralysis we inject a high dose of rocuronium $(1 \mathrm{mg} / \mathrm{Kg})$ with the great advantage of the possible antagonism assured by sugammadex in case of difficult of intubation. In international literature there is a constant debate about the safety of suxamethonium. ${ }^{6}$ In the review by Rawicz et al., the reasons for continuing the use of suxamethonium, in the opinion of Martin Rawicz, are the following: i) there is no other drug that can provide both rapid and ultra-short muscle relaxation; ii) the neuromuscular block produced by suxamethonium does not require reversal; iii) metabolism of suxamethonium is organ independent; iv) suxamethonium can be a lifesaving drug in case of severe laryngospasm; v) in the vast majority of patients, suxamethonium-associated side effects are never observed or are of minimal clinical significance.

Barbara Brandom provides the following reasons for completely stopping the injection of this relaxant: i) suxamethonium increases muscle stiffness; ii) nondepolarizing neuro- muscular blockers can produce better intubating conditions; iii) suxamethonium produces muscle pain; iv) suxamethonium can produce myoglobinuria which can be a threat to renal function; v) evaluation of the causes of myoglobinuria can be costly; vi) diagnosis of occult myopathy can be difficult; vii) suxamethonium has caused fatal hyperkalemic cardiac arrests in infants and children; viii) suxamethonium can increase intracranial pressure.

Anyway, we think that the antagonism assured by sugammadex represents an additional safety factor together with the use of rocuronium and a larger use of these drugs should be recommended.

Total intravenous anesthesia (TIVA) is another effective and safe technique to manage anesthesia during many surgical procedures and may be applied also to pediatric patients with airway foreign body removal for which TIVA can assure a spontaneous ventilation. Propofol and remifentanli are he drugs most commonly used. In patients affected by myopathy, the TIVA represents a valid alternative to avoid the risk of malignant hyperthermia caused by halogenated anesthetics.

Regional anesthesia is representing a large field developing in the pediatric population with particular reference to the ultrasound techniques. Epidural caudal block is one of the most common regional blocks. It's a simple procedure and the use of the latest local anesthetics (ropivacaine and levobupivacaine) reduces the risk of cardiotoxicity and neurotoxicity. The ultrasound transversus abdominis plane (TAP) block has more recently become a very interesting technique also in pediatric patients together with ultrasound peripheral nerve blocks both single shot and continuous infusion of local anesthetics. ${ }^{7,8}$

Allergic reactions are very rare nowadays in our operating rooms. Latex allergies can occur and also be prevented by using fewer latexcontaining items as well as inquiring about this problem during the pre-anesthetic examination. Using latex-free equipment is especially advisable when giving anesthesia to patients who will be operated on again in the future. Among the drugs used in anesthesia the most capable of eliciting an allergic reaction are the antibiotics and, albeit very rarely, muscle relaxants. If a patient had an inexplicable shock during the induction of a previous anesthetic, it is advisable to use a different muscle relaxant. The clinical manifestations of anaphylaxis are the consequences of the immediate as well as ongoing release of preformed mediators from mast cells and basophils. ${ }^{9}$ The Ring and Messmer clinical severity scale, cited by Dewatcher et al., distinguishes the following reaction levels: Grade 1. Cutaneous mucous signs: erythema, urticaria with or without angioedema; Grade 2. Moderate multivisceral signs: cutaneous mucous signs \pm hypotension \pm tachycardia \pm dyspnea \pm gastrointestinal disturbances; Grade 3 . Lifethreatening mono-or multivisceral signs: cardiovascular collapse, tachycardia, or bradycardia \pm cardiac dysrythmia \pm bronchospasm \pm cutaneous mucous signs \pm gastrointestinal disturbances; Grade 4. Cardiac arrest.

The overall incidence of perioperative anaphylaxis is estimated at 1 in 10-20,000 anesthetic procedures. It is noteworthy that in some cases bradycardia may be a life-protecting adaptive mechanism that allows the ventricle to fill despite hypovolemia. ${ }^{9}$ Then the treatment with atropine must be simultaneous with epinephrine and fluids; otherwise giving only atropine can result in cardiac arrest. As a matter of fact epinephrine and an expansion of intravascular volume are the key points in the perioperative management of anaphylaxis. ${ }^{9}$ In grade 1 reactions there is no indication of epinephrine (in these cases we only observe the patient, report the reaction in the anesthesia record and do not give any treatment, for the great part of these are self limited). In grade 2 reactions it is necessary to inject titrated boluses (10-20 mcg) of epinephrine, whereas in grade 3 reactions boluses of $100-200 \mathrm{mcg}$ are required; only in grade 4 reactions it is necessary to give $1-3 \mathrm{mg}$ intravenously. It is important to quickly differentiate between grade 1, 2, 3 or 4 reactions because unnecessarily high doses of epinephrine can disrupt the cardiac microvasculature resulting in myocardial disfunction. Stress-induced cardiomyopathy, or takotsubo cardiomyopathy, is described after anaphylactic shock and it is unclear if the cardiac problem stems from the anaphylactic shock (coronary artery spasms by anaphylactic mediators) or from the overzealous administration of epinephrine. ${ }^{10,11}$ There are rare cases of anaphylactic shock refractory to cathecolamines: in these situations arginine vasopressin may be an alternative through its vasoconstrictive effects mediated by nonadrenergic vascular V1 receptors. ${ }^{9}$ Another innovative therapy is methylene blue because it interferes with nitric-oxide mediated vascular smooth muscle relaxation. The use of epinephrine can be a source of complications. The standard dose is $0.01-0.02 \mathrm{mg} / \mathrm{Kg}$ intravenously. Sometimes doctors are overzealous, using a high dosage due to their impatience in the treatment of an acute condition. According to recent reviews high doses can worsen a patient's post resuscitation hemodynamic condition by causing increased myocardial oxygen demand, ventricular ectopy, hypertension and myocardial necrosis. High doses do not improve survival and may be associated with a worse neurological outcome. ${ }^{12}$ In particular, we think it is not wise to use high dose epinephrine in conditions such as hypovolemic shock in which the optimization of the preload is the first-line treatment. 
Cathecolamines can also temporarily disrupt the cardiac microvasculature, resulting in myocardial dysfunction. ${ }^{10}$

Mechanical ventilation can be a source of complications, first of all pulmonary barotrauma and volutrauma. Usually we start mechanical ventilation with a very low tidal volume; then we slowly increase it until we observe the chest moving and hear gas entering the lungs with the stethoscope. We also check the monitor, looking for an acceptable capnographic curve and a peak inspiratory pressure in the airways no higher than $15-20 \mathrm{~cm} \mathrm{H}_{2} \mathrm{O}$. We fear volutrauma-barotrauma more than a moderate hypercapnia, so we do not try to obtain normocapnia if this entails a dangerous increase in peak inspiratory pressure. According to recent studies summarized by Curley et al.,13 mechanical ventilation at even clinically relevant low tidal volumes and for relatively short periods of time may activate an inflammatory response in the lung. So, if low stretch mechanical ventilation initiates an inflammatory response, it is mandatory to avoid any form of baro-or volutrauma in the lungs, especially in newborns and children.

Fluid therapy is part of the job of the anesthesiologist. We advise to try always to avoid hypovolemia; so, in children who are beyond the neonatal age, and in absence of particular problems such as heart disease with retention of fluid, we are in favor of a liberal administration of saline. The child is able to urinate the excess administration but is unable to create water if we infuse an insufficient amount of fluids. If the child arrives in the operating room with a hyperalimentation infusion, it is better not to discontinue it, for the risk of metabolic derangement secondary to the abrupt stops. When giving anesthesia to a child with a functioning intravenous infusion, it is advisable to remember Poiseuille's law: very narrow and very long catheters can be suitable for parenteral nutrition and drug therapy in the ward but are not reliable in the operating room for rapid administration of drugs, fluids and blood. So we think it is better to place a normal intravenous cannula, even if the child already has a catheter for parenteral nutrition. Infusion pumps can be a source of complication: the pump will continue to push the fluid even if the cannula is outside a vein so the site in which the cannula is positioned must be constantly inspected.

Careful hemodynamic monitoring is required and the use of invasive or less invasive techniques is debated. Usually we prefer less invasive or noninvasive techniques. We have recently published an interesting original article about the hemodynamic effects of levobupivacaine after pediatric caudal anesthesia evaluated by transesophageal doppler (Pediatric CardioQ). We demonstrated that no differences in hemodynamic parameters could be detected between balanced general anesthesia with either caudal levobupivacaine or remifentanil infusion in pediatric patients undergoing genitourinary surgery. Hemodynamic monitoring by the use of transesophageal doppler is easy to perform and is completely noninvasive. Many hemodynamic parameters (cardiac output, indexed cardiac output, peak velocity, flow time corrected (FTc), stroke volume, indexed stroke volume, etc.) may be evaluated, especially regarding the loss of fluids during major surgical procedures. This method allows us to properly assess blood loss, reducing the risk of invasive techniques. The pediatric CardioQ may also applied in neonates and infants. ${ }^{14}$

\section{Airway management}

Tracheal intubation is not always necessary during minor surgery and often exposes patients to risks of airway irritation and laryngospasm. In our clinical experience, we make extensive use of laryngeal mask airway and proseal laryngeal mask airway also in neonates in combination with regional anesthesia and ultrasound regional block without the administration of muscle relaxants. The laryngeal mask airway should be avoided in non-fasting patients and in case of important bleeding. We have developed and published in literature a novel technique for the concurrent use of laryngeal mask and transesophageal doppler that allows to ventilate and perform hemodynamic monitoring in a completely noninvasive procedure. 15,16

The emergence from general anesthesia can be a risky moment. First we must be certain that the patient has regained a normocapnic state so we disconnect the mechanical ventilator and, without stimulating the child with suction or other methods, we wait for the return of spontaneous ventilation; then we inject small increments of neostigmine (up to a maximum of $0.05 \mathrm{mg} / \mathrm{Kg}$ ), adding atropine only if bradycardia ensues. We then remove the endotracheal tube only when the child is fully awake, opens his or her eyes, and makes purposeful movements. For at least 1 hour after the end of the procedure, the child must be looked after in an environment (recovery room) in which monitoring equipment and trained personnel are available to intervene in case of respiratory depression, laryngospasm and so on. Newborns, especially if premature, must be looked after with special care. Their ventilatory response to $\mathrm{CO} 2$ is blunted, and hypoxia further blunts this response. Residual anesthetic drugs can depress the ventilation and newborns may be unable to react to hypoxia-hypercapnea, with a very dangerous mix of factors that can lead to apnea. Awareness, or the unwanted recall of intraoperative events during general anesthesia, is very rare in pediatric anesthesia. A recent study on 1784 children found possible/probable awareness only in $0.8 \%$ of the cases. 17 The authors believe that this problem is underrecognized and undertreated: awareness may be particularly underrecognized in children who may be reluctant or unable to describe their intraoperative memories. In their study, an endoscopic procedure was the only factor found to pose a higher risk for awareness.

In endoscopic procedures the anesthesiologists tend to arrange for a lighter plane of anesthesia as there are not painful stimuli. We also perform this way! The authors conclude warning that a child's report of intraoperative recall should not be readily dismissed but addressed with appropriate follow-up. Another study on long-term post-traumatic effects of intraoperative awareness, ${ }^{18}$ although on a very small number of children, led to the conclusion that awareness is a rare event in children, and post-traumatic stress is exceedingly rare, but possible.

\section{Problems unrelated to the anesthesia}

The following disorders can be erroneously attributed to:

Dilated cardiomyopathies represent a heterogenous group of myocardial disorders that result in ventricular dilation and impaired systolic function. ${ }^{19}$ Ventricular tachycardia, torsade de pointes and ventricular fibrillation can occur. Besides primary dilated cardiomyopathy, which is genetically transmitted, there are some secondary forms that can be present without our knowing it. They can follow infections, environmental and toxin exposures, autoimmune reactions, nutritional deficiencies, metabolic disorders, and the treatment of cancer with anthracycline-derived chemotherapeutic agents and/or radiation. ${ }^{19}$ So a patient can suffer a malignant arrhytmia during the anesthesia due to a subclinical form of cardiomyopathy.

Hypertrophic cardiomyopathy is characterized by left ventricle hypertrophy, diastolic dysfunction, obstruction to left ventricle outflow and in some cases sudden cardiac death in young healthy subjects. ${ }^{19}$ Clinical predictors can be sudden cardiac death in a first-degree relative an unexplained syncope, but the disease can be silent, and sudden cardiac death can occur in the operating room instead of the football arena.

Arrhythmogenic right-ventricular dysplasia is a genetic disease characterized by a fatty fibrous replacement of the right ventricle. ${ }^{19}$ The process of replacing normal myocardium 
with fatty and fibrous tissue predisposes patients to right ventricle dysfunction and ventricular arrhythmias. Sudden cardiac death may be the initial presentation of the disease. In all cases of sudden, unexplained cardiac problems during or after anesthesia it is advisable to perform a myocardial biopsy and/or cardiac magnetic resonance imaging to assess the existence of such cardiac disease that can be responsible for the complications.

Myocarditis, characterized by an inflammatory cellular infiltrate of the myocardium, ${ }^{20,21}$ with necrosis and/or degeneration of myocites, can result from the following: i) viral infections; ii) infections from bacteria, fungi, parasites; iii) hypersensitivity reactions; iv) autoimmune disorders; v) pharmaceuticals (acetazolamide, amphotericin B, anthracyclines, cephalosporins, cocaine, cyclophosphamide, digoxin, diureticsm, dobutamine, indomethacin, isoniazid, methyldopa, neomercazole, penicillin, phenylbutazone, phenytoin, sulfonamides, tetracycline, tricycle antidepressants).

Clinical manifestations of myocarditis can be erroneously attributed to the anesthesia. Manifestations range from subclinical disease to serious cardiac problems: atrial or ventricular arrhythmias, infarction, sudden death. ${ }^{21}$ Children often have a fulminant presentation. The myocardial biopsy (which can be risky) and the magnetic resonance imaging (non invasive and devoid of risk) are important for the diagnosis. The treatment of myocarditis includes the administration of angiotensinreceptor blockers, beta blockers and diuretics. ${ }^{21}$ In some cases an implantable cardioverter-defibrillator is necessary. Sometimes immunological therapies are used such as immunoglobulins, corticosteroids, cyclosporine. In some cases an antecedent flu-like illness or gastroenteritis may precede the myocarditis. ${ }^{20}$

But what do recent reviews on the topic of complications in pediatric anesthesia indicate as the most common cause? Aleksic et al. ${ }^{22}$ in Serbia highlight that the risk of complications in children is increased if anesthesia is administered in the following situations: i) is not administered by a pediatric anesthetist; ii) is administered by a resident supervised by an anesthesiologist who is involved with other cases.

Even before reading this paper, we were positively convinced that each senior anesthesiologist should take care of one case and one resident!

The Serbian colleagues also noted that the sitting position carries a great risk of complications (venous air embolism, bradycardia, arrhythmia, bronchospasm, hypoxia). Kakavouli et al. ${ }^{23}$ in New York reported that intraoperative adverse events were more common in patients with a higher ASA status or a younger age, and that respiratory adverse events were most common. Pediatrics reported adverse events incidence was comparable for non operating room anesthesia and operating room anesthesia. We acknowledge the data of the American colleagues but we think that, when possible, the anesthesia should be given in the safer environment of the operating room: it is better to move the doctor who is going to perform a procedure (endoscopy, placement of central intravenous lines) than to move the child. Of course there are procedures (radiotherapy, magnetic resonance imaging) for which the patients have to be moved. Ahmed et al. ${ }^{24}$ in Pakistan made an analysis of anesthesia-related complications at a university teaching hospital over 15 years. They found that adverse events were provoked by inadequate ventilation after extubation, underreplacement of fluids, bradycardia after suxamethonium, two vaporizers open simultaneously, and they understandably stressed the importance of the presence of an experienced anesthesiologist. Sanders and Davidson,25 from Australia, raised the problem of a possible anesthetic-induced neurotoxicity in the neonate, citing various experimental works in newborn animals. Their conclusions are as follows: we do not know how possible a neurotoxic effect is on the developing brain pain is associated with poor outcome, so it is not wise to reduce the dosage of anesthetics and analgesics for the fear of toxicity it is advisable to delay the procedures that require general anesthesia, if they are not of vital importance and urgent. But the authors state as we are unsure of the age when exposure is relevant, we cannot provide any firm recommendation on how long surgery should be delayed. Stratmann, ${ }^{26}$ citing various studies performed in rodents, observes that all intravenous and volatile anesthetics are GABA-ergic, and GABA is excitatory in the immature brain. So the authors cited hypothesize a possible maintenance non convulsive seizures, especially with a clinically relevant concentration of sevoflurane, distinct from the well-known sevoflurane emergence seizures (which we observe very rarely, almost never). These maintenance seizures are experimentally prevented by bumetanide, a loop diuretic that inhibits the chloride transporter NKCC1. Stratmann acknowledges that these studies have discovered a previously unknown mechanism by which GABA-ergic anesthetics may harm the neonatal brain but until new data from clinical studies are available, he is sure that no change in clinical practice is justified. In a recent clinical commentary Perouanski and Hemmings state that anesthetics can effect gene expression, protein synthesis and processing, and cellular function in poorly understood ways that provide plausible biochemical substrates for durable long term effects in a num- ber of tissues. ${ }^{27}$ The authors think that, given the profound alterations of central nervous system function induced by general anesthesia, some degree of long-lasting effects is possible; however they acknowledge that studies in human subjects are very difficult (the aforementioned studies were performed in rodents). So far, the few clinical trials have not proven or excluded a link between anesthesia and long term neuralgic damage. Probably, in our opinion, the best way for preventing a long-term toxicity is to use a combination of drugs and not a single agent.

Before closing this review we want to remember the existence of sudden infant death syndrome. It is the sudden death of an infant or young child, which is unexpected by history, and in which a thorough postmortem examination fails to demonstrate an adequate cause of death. 28 The syndrome has a peak incident at 2-4 months of age. Most of the causes are unknown but predisposing factors are inherited disorders of fatty acid oxidation, genetic cardiac channello-pathies, and infections (seemingly trivial) that can precipitate a lethal cytokine cascade or toxic response. ${ }^{28}$ On these predisposing factors an exogenous stressor can superimpose, and determine the death of the infant. The stressors include the prone sleeping position, bedclothes covering the head, bed sharing, high ambient temperature, exposure to parental smoking. Prenatal exposure to alcohol and smoke can alter the neurotransmitters and predispose the infant to sudden death. After an apparently uneventful general anesthesia, if an infant belongs to these risk groups, a sudden death can happen, and can be attributed to the anesthesia.

In addition to all the foregoing we want to remember the many possible complications that follow the loss of sleep and fatigue of the anesthesiologist.29 Many studies demonstrate that performance after 16 hours of wakefulness is comparable to a blood alcohol level of $0.1 \%$ ! Individual physicians need to think of sleep loss as impairment, and comparable to alcohol impairment.

\section{References}

1. Spaeth JP, Kurth CD. The extremely premature infant (micropremie). In: Cote CJ, Lerman J, Todres ID, eds. A practice of anesthesia for infants and children. Philadelphia: Saunders; 2009.

2. Simon BA, Moody EJ, Johns RA. Therapeutic gases oxygen, carbon dioxide, nitric oxide and helium. In: Brunton L, Lazo J, Parker K, eds. Goodman and Gilman's, the pharmacological basis of therapeutics. New York: Mc Graw Hill; 2006. 
3. De Hert SG, Preckel B, Schalack WS. Update on inhalational anesthetics. Curr Opin Anaesthesiol 2009;22:491-5.

4. Evers AS, Crowder CM, Balser JR. General anesthetics. In: Brunton L, Lazo J, Parker K, eds. Goodman and Gilman's, the pharmacological basis of therapeutics. New York: Mc Graw Hill; 2006.

5. Naguib M, Brull SJ. Update on neuromuscular pharmacology. Curr Opin Anaesthesiol 2009;22:483-90.

6. Rawicz M, Brandom B, Wolf A. The place of suxamethonium in pediatric anesthesia. Pediatr Anesth 2009;19:561-70.

7. Galante D. Ultrasound needle guidance in neonatal and infant caudal anesthesia. Pediatr Anesth 2008;18:1233-4.

8. Galante D. Regional caudal blockade in a pediatric patient affected by the Joubert syndrome. Acta Anaesthesiol Scand 2009;53:693-4.

9. Dewachter P, Mouton-Faivre C, Emala CW. Anaphylaxis and anesthesia: controversies and new insights. Anesthesiology 2009; 111:1141-50.

10. Bainbridge D, Cheng D. Stress-induced cardiomyopathy in the perioperative setting. Can J Anaesth 2009;56:397-401.

11. Suk E, Kim D, Kweon T et al. Stressinduced cardiomyopathy following cephalosporin-induced anaphylactic shock during general anesthesia. Can J Anesth 2009;56:432-6.

12. Berg R, Mijasaka K, Rodriguez-Nunez A. Cardiopulmonary resuscitation. In: Nichols DG, ed. Rogers' textbook of pedi- atric intensive care. Philadelphia: Lippincott Williams \& Wilkins. 2008.

13. Curley GF, Kevin LG, Laffey JG. Mechanical ventilation - Taking its toll on the lung. Anesthesiology 2009;111:701-3.

14. Galante D, Pellico G, Meola S, et al. Hemodynamic effects of levobupivacaine after pediatric caudal anesthesia evaluated by transesophageal Doppler. Pediatr Anesth 2008;18:1066-74.

15. Galante D. Transesophageal Doppler probe and proseal laryngeal mask airway. A New technique for probe insertion in pediatric anesthesia. Anesth Analg 2008;107:348.

16. Galante D. The Proseal laryngeal mask airway and the transesophageal Doppler probe: the TED-PLMA Technique. Can J Anaesth 2011;58:1144-5.

17. Malviya S, Galinkin J, Bannister C et al.: The incidence of intraoperative Awareness in children: Childhood awareness and recall evaluation. Pediatr Anesth 2009;19:1421-7.

18. Phelan L, Stargatt R, Davidson AJ. Longterm post-traumatic effect of intraoperative awareness in children. Pediatr Anesth 2009;19:1152-6.

19. Jefferies JL, Denfield SW, Dreyer WJ. Heart failure in infants and children: cardiomyopathy. In: Nichols DG, ed. Rogers' textbook of pediatric intensive care. Philadelphia: Lippincott Williams \& Wilkins; 2008.

20. Breinholdt JP, Nelson DP, Towbin DP. Heart failure in infants and children: myocarditis. In: Nichols DG, ed. Rogers' textbook of pediatric intensive care. Philadelphia: Lippincott Williams \& Wilkins; 2008

21. Cooper LT. Myocarditis. New Engl J Med 2009;360:1526-38.

22. Aleksic V, Radulovic D, Milakovic B, et al. A retrospective analysis of anesthesiologic complications in pediatric neurosurgery. Pediatr Anesth 2009;19:879-86.

23. Kakavouli A, Li G., Carson M, et al. Intraoperative reported adverse event in children. Pediatr Anesth 2009;19:732-9.

24. Ahmed A, Alim A, Khanm, Khan F. Perioperative cardiac arrests in children at a university teaching hospital of a developing country over 15 years. Pediatr Anesth 2009;19:581-6.

25. Sanders RD, Davidson A. Anestheticinduced neurotoxicity of the neonate: time for clinical guidelines? Pediatr Anesth 2009;19:1141-6.

26. Stratmann GS. Aneseirure: is the neonatal brain asleep? Anesthesiology 2010;112: 527-9.

27. Perouansky M, Hemmings $H$. Neurotoxicity of general anesthetics: cause for concern? Anesthesiology 2009;111:1365-71.

28. Kinney HC, Thach BT. The sudden infant death syndrome, New Engl J Med 2009; 361:795-805.

29. Flynn TC. Sleep and fatigue. In: Lobato E, Gravensteinn N, Kirby RR, eds. Complications in anesthesiology. Philadelphia: Lippincott Williams \& Wilkins; 2008. 